\section{Diabetes Mellitus gestaCiONAL}

Autoria: Sociedade Brasileira de Endocrinologia e Metabologia

Elaboração final: 20 de junho de 2006

Participantes: Miranda PAC, Reis R

Descrição do método de coleta de evidência: Revisão da literatura

\section{Graus de recomendação e força de evidência:}

A: Estudos experimentais ou observacionais de melhor consistência.

B: Estudos experimentais ou observacionais de menor consistência.

C: Relatos de casos (estudos não controlados).

D: Opinião desprovida de avaliação crítica, baseada em consensos, estudos fisiológicos ou modelos animais.

\section{Objetivo:}

Fornecer as principais recomendações relacionadas à identificação e ao manuseio da gestante com diabetes.

\section{Conflito de interesse:}

Nenhum conflito de interesse declarado.

\section{Introdução}

Diabetes mellitus gestacional (DMG) é definido como qualquer nível de intolerância a carboidratos, resultando em hiperglicemia de gravidade variável, com início ou diagnóstico durante a gestação. Sua fisiopatologia é explicada pela elevação de hormônios contra-reguladores da insulina, pelo estresse fisiológico imposto pela gravidez e a fatores predeterminantes (genéticos ou ambientais). $\bigcirc$ principal hormônio relacionado com a resistência à insulina durante a gravidez é o hormônio lactogênico placentário, contudo, sabe-se hoje que outros hormônios hiperglicemiantes como cortisol, estrógeno, progesterona e prolactina também estão envolvidos.

A incidência de DMG é de 3\% a 7\%, variando de acordo com a população estudada e com os critérios diagnósticos utilizados. Nos Estados Unidos, são diagnosticados 135.000 novos casos por ano, tendo uma prevalência de I,4\% a 2,8\%, nas populações de baixo risco e de 3,3\% a 6,1\%, nas populações de alto $\operatorname{risco}^{\prime}(\mathbf{A})$.

No Brasil, estima-se prevalência de 2,4\% a 7,2\%, dependendo do critério utilizado para o diagnóstico²(A).

Devido a implicações éticas, estudos aleatorizados a respeito do DMG são raros e, sendo assim, as recomendações desta diretriz serão sustentadas, na maior parte das vezes, por estudos com outro tipo de desenho.

\section{Por que diagnosticar o DMG?}

A gestante portadora de DMG não tratada tem maior risco de rotura prematura de membranas (OR 10,07), parto prétermo (OR 6,42), feto com apresentação pélvica $(O R$ 3,47) e feto macrossômico $(\mathrm{OR} 2,42)^{3}(\mathbf{A})$. Há também risco elevado de pré-eclâmpsia nessas pacientes, com OR 2,26 ${ }^{4}(\mathbf{D})$.
Com relação ao feto, além da macrossomia, o risco para o desenvolvimento de síndrome de angústia respiratória, cardiomiopatia, icterícia, hipoglicemia, hipocalcemia, hipomagnesemia e policitemia com hiperviscosidade sangüínea, encontra-se fortemente aumentado ${ }^{5,6}(\mathbf{D})$.

$\bigcirc$ resultado perinatal está diretamente relacionado ao controle metabólico materno, com evidência de 52,4\% de macrossomia, 14,3\% de óbito fetal e 8,2\% de má formações em gestantes com controle metabólico não adequado, caracterizado por média glicêmica superior a 130 mg/dl no terceiro trimestre $^{7}(\mathbf{C})$.

\section{Como diagnosticar DMG?}

O diagnóstico envolve duas fases distintas - rastreamento e confirmação diagnóstica. Os critérios e testes utilizados para o rastreamento e diagnóstico do DMG são controversos, pela existência limitada de estudos comparativos.

teste inicial recomendado para a triagem de DMG é a dosagem da glicemia plasmática I h após teste oral com 50g de dextrosol, devendo ser realizado entre a $24^{\mathrm{a}}$ e $28^{\mathrm{a}}$ semanas de gestação. São aceitos como valores de corte tanto 140 mg/dl quanto $130 \mathrm{mg} / \mathrm{dl}$, com cerca de $80 \%$ e $90 \%$ de sensibilidade, respectivamente $(\mathbf{D})$.

A dosagem da glicemia plasmática em jejum também pode ser utilizada como rastreamento e diagnóstico de DMG. A associação glicemia de jejum (GJ) + fator de risco (FR) é método alternativo de rastreamento, orientado pelo Ministério da Saúde do Brasil, considerando a praticidade e o baixo custo, pois a GJ é exame de rotina e a investigação de riscos para DMG é obrigatória na anamnese da primeira consulta no prénatal ${ }^{8}(\mathbf{D})$. Durante o primeiro trimestre de gestação, os níveis glicêmicos tendem a valores mais baixos, contudo mais estudos são necessários para a determinação de valores de referência para esta fase. Como não existe uma definição clara, valores acima de 100 mg/dl devem ser considerados alterados. A partir da $24^{a}$ semana de gestação, a glicemia de jejum com valores acima de $85 \mathrm{mg} / \mathrm{dl}$ pode ser considerada como rastreamento positivo. Este parâmetro, por sua vez, apresenta um VPN (valor preditivo negativo) de 97\%, porém baixa sensibilidade e especificidade $^{9}(\mathbf{B})$. Valores acima de $110 \mathrm{mg} / \mathrm{dl}$ confirmam o diagnóstico de DMG, em qualquer fase de gestação.

Resultados anteriores observados em nosso meio demonstraram equivalência estatística no rastreamento feito pelo Teste Oral de Tolerância à Glicose (TOTG) $50 \mathrm{~g}$ e pela associação Gl $+\mathrm{FR}^{10}(\mathbf{C})$. Estudo recente ratificou tais resultados e evidenciou semelhança estatística entre os dois métodos (TOTG 50g e GJ+FR) nos índices de sensibilidade (S) (86,4 e 76,9\%), valor preditivo negativo (VPN) $(98,7$ e 98,9\%), razão de probabilidade negativa (RPN) $(0,3$ e 0,3$)$ e resultados falso-negativo (FN) (15,4 e 23,1\%), respectivamente, comparados ao teste diagnóstico (TOTG $100 \mathrm{~g})^{\prime \prime}(\mathbf{B})$. Estudo comparando os critérios diagnósticos adotados pela ADA (American Diabetes 
Association) com parâmetros adotados pela OMS (Organização Mundial de Saúde), para o teste oral de tolerância a glicose com $75 \mathrm{~g}$ de dextrosol, concluiu que os últimos são mais sensíveis, sem alterar o intervalo de confiança para detecção de risco de complicações relacionadas ao $\operatorname{DMG}^{2}(\mathbf{A})$. Colaborando com este dado, outro estudo, utilizando os critérios da OMS, demonstrou um alto índice de complicações em pacientes portadoras de DMG não $\operatorname{tratadas}^{3}(\mathbf{A})$. Estudos observacionais demonstraram que grávidas com apenas um valor alterado, segundo os critérios de Carpenter e Coustan, estão mais propensas a complicações do que pacientes controle ${ }^{12}(\mathbf{D})$.

O TOTG lo0g é mau preditor da macrossomia fetal $^{13,14}(\mathbf{C})$. O perfil glicêmico (PG) é teste utilizado no diagnóstico e no controle de tratamento do DMG e da hiperglicemia diária, padronizado pela avaliação das glicemias plasmáticas maternas, a cada duas horas, por um período de 12 horas (diagnóstico) ou 24 horas (tratamento). Os limites de normalidade são valores abaixo de 90 mg/dl, no jejum e inferiores a $130 \mathrm{mg} / \mathrm{dl}$, nas pósprandiais. A resposta, normal ou alterada, do PG é independente do resultado do TOTG I00g e pode estar alterada de forma isolada, confirmando a hiperglicemia diária (grupo IB de Rudge), ou associada à resposta anormal ao TOTG I00g, diagnosticando o DMG (grupo IIB de Rudge) ${ }^{15}(\mathbf{C})$. Este grupo representa 13,8\% da população de gestantes com rastreamento positivo para DMG, com percentual elevado de macrossomia e morte perinatal comparável às gestações associadas ao diabetes e 10 vezes maiores que a gestação normal' ${ }^{16}(\mathbf{B})$.

Sob a luz destas informações, acreditamos que adotar critérios mais abrangentes, com a intenção de evitar ao máximo falso-negativos durante a pesquisa diagnóstica, é a melhor conduta no DMG.

\section{Quais pacientes devem ser pesquisadas?}

Vários são os fatores de risco pré-gestacionais e gestacionais relacionados ao desenvolvimento do DMG, que devem ser usados como guia para atenção individualizada à paciente. A triagem em todas as gestantes é o mais recomendado, pois quando nos baseamos em fatores de risco, diminuímos a necessidade de confirmação diagnóstica'2(D).

A $\operatorname{ADA}^{2}(\mathbf{A})$ orienta os seguintes limites para indicação de insulinoterapia associada à dieta, o que, indiretamente, caracteriza as metas para as glicemias plasmáticas no controle de tratamento:

- Glicemia de jejum $\leq 105 \mathrm{mg} / \mathrm{dl}$

- Pós-prandial de lhora $\leq 155 \mathrm{mg} / \mathrm{dl}$

- Pós-prandial de 2 horas $\leq 130 \mathrm{mg} / \mathrm{dl}$

manual de orientação da FEBRASGO, Diabete e hipertensão na gravidez, é mais rígido quanto ao controle glicêmico materno. Preconiza o limite de 90 mg/dl para o jejum e de 130 $\mathrm{mg} / \mathrm{dl}$ para qualquer pós-prandial, o que refletiria em média glicêmica diária inferior a $120 \mathrm{mg} / \mathrm{dl}^{17}(\mathbf{D})$.

\section{Como tratar o diabetes gestacional?}

Revisão sistemática sobre a eficácia da insulina na prevenção da macrossomia em fetos de mães diabéticas gestacionais, incluindo |28| gestantes, mostrou benefícios da insulina comparada à dieta. Porém, os achados (redução de risco 0,098; IC: 0, I68 a 0,028) indicam a necessidade de novos estudos controlados, pela heterogeneidade na literatura ${ }^{18}(\mathbf{A})$.

\section{Quais são as metas para o tratamento?}

A - Glicose plasmática em jejum < 105 mg/dl.

ou

Glicose de jejum em sangue total $<95 \mathrm{mg} / \mathrm{dl}$.

B - Glicose plasmática Ih pós-prandial < |55mg/dl

ou

Glicose I h pós-prandial em sangue total < |40mg/dl

\section{Tratamento alimentar}

Dietas abaixo de $1200 \mathrm{Kcal} /$ dia ou com restrição de mais de $50 \%$ do metabolismo nasal não são recomendadas, pois estão relacionadas com desenvolvimento de $\operatorname{cetose}^{19}(\mathbf{A})$.

A grávida portadora de DMG deve fazer aproximadamente seis refeições por dia, sendo três principais e três lanches. $\bigcirc$ lanche noturno é importante para evitar a cetose durante o sono $^{5}(\mathbf{D})$.

Grávidas obesas devem ser submetidas a leve restrição calórica, com total de $25 \mathrm{Kcal} / \mathrm{kg}$ de peso atual por dia. Grávidas com peso normal devem ser orientadas a ingerir um total calórico diário em torno de $30 \mathrm{Kcal} / \mathrm{kg}$ de peso e grávidas de baixo peso $35 \mathrm{Kcal} / \mathrm{kg}$. Nos $2^{\circ}$ e $3^{\circ}$ trimestres de gestação, deve-se adicionar $300 \mathrm{Kcal}$ por dia. $\bigcirc$ valor calórico total deve ser bem distribuído durante o dia, com I 5\% no café da manhã, I0\% na colação, 30\% no almoço, 10\% no lanche da tarde, 25\% no jantar e $10 \%$ na $\operatorname{ceia}^{20}(\mathbf{D})$.

A distribuição sugerida dos nutrientes é de $40 \%$ a $50 \%$ de carboidratos, $25 \%$ a $30 \%$ de proteínas e $25 \%$ a $30 \%$ de gorduras.

\section{Atividade física}

Atividade física leve a moderada, em pacientes sem contraindicações clínicas ou obstétricas, contribui para a redução e o controle da glicemia.

\section{Insulinoterapia}

Insulinização é indicada quando:

- Jejum > 90 mg/dl e qualquer pós-prandial > 130 mg/dl na vigência de dieta exclusiva, com dose inicial calculada pela seguinte fórmula ${ }^{9}(\mathbf{D})$ :

$$
\frac{\text { Jejum }-90}{4}
$$

- Após uma semana de adoção das medidas dietéticas sem atingir alvo de tratamento; 
- Parâmetros ultra-sonográficos indicativos de macrossomia ou polidrâminio, mesmo na vigência de parâmetros laboratoriais adequados.

Deve-se dar preferência à insulina humana em detrimento da animal, pelo fato da primeira ser menos imunogênica, prevenindo a formação de anticorpos, que ultrapassam a barreira placentária.

Apesar de alguns estudos demonstrarem que a insulina lispro não é encontrada na circulação fetal e do uso clínico esporádico em gestantes, ainda não existem evidências que embasam esta conduta ${ }^{5,21}$ (D). Não existem trabalhos sobre o uso de insulina aspart ou glargina em gestantes.

\section{Anti-hiperglicemiantes orais}

Não existem evidências que justifiquem ou embasem o uso de anti-hiperglicemiantes durante a gestação. Entretanto, um estudo sobre o uso do gliburide, sulfoniluréia de segunda geração, que não cruza a placenta, demonstrou ação comparável à insulina e necessidade de substituição por insulina em menos de 10\% das diabéticas randomizadas ${ }^{22}(\mathbf{A})$. $\bigcirc$ uso desta droga em gestantes diabéticas ainda não foi aprovado pelo Food and DrugsAdministration (FDA), sendo necessários mais estudos, em maior número de gestantes, para estabelecer a segurança deste tipo de terapia. Assim, mantém a orientação de se usar insulina, e não anti-hiperglicemiantes orais, durante a gestação ${ }^{5}(\mathbf{D})$.

\section{Atenção pré-natal}

São pontos de destaque na assistência pré-natal das portadoras de DMG, a freqüência das consultas, o controle metabólico materno e a avaliação do bem-estar fetal $\left.\right|^{17}(\mathbf{D})$.

- As consultas de pré-natal devem ser quinzenais, do diagnóstico de DMG até a 32a semana e, daí em diante, semanais até o parto;

- $\bigcirc$ controle glicêmico materno coincide com as consultas de pré-natal e é realizado pelo perfil glicêmico (PG) de 24 horas, nas gestantes usuárias de insulina e de 12 horas, nas controladas com dieta e exercício físico;

- Deve ser realizado um ultra-som no primeiro trimestre para datar a idade gestacional, um segundo entre a $24^{\mathrm{a}} / 25^{\mathrm{a}}$ semanas para avaliação da morfometria fetal e, a partir da $30^{a}$ semana, deve ser mensal para avaliação da biometriadesenvolvimento fetal, índice de líquido amniótico (ILA) e grau placentário, com Doppler das artérias umbilical e cerebral média, de preferência, a cada quinze dias;

- Cardiotocografia anteparto - a partir da 28a/30a semana de gestação, semanal, nas gestantes seguidas em ambulatório e diária, nas gestantes durante as internações.

\section{Atenção peri e pós-parto}

Não existem recomendações específicas para via de parto, sendo esta determinada pelas condições obstétricas específicas da paciente. Por existir um risco maior de desenvolvimento de macrossomia após a $38^{a}$ semana, é recomendado que a gestação não se prolongue além deste período.

No período pré-parto, a meta do controle glicêmico deve estar entre 80 e 120 mg/dl. $\bigcirc$ controle da hiperglicemia é de grande importância, pois a maioria das complicações fetais está relacionada à elevação da glicemia materna no pré e peri-parto.

Gestantes que não usaram insulina durante a gestação, geralmente, não necessitam de cuidados especiais durante o parto. A partir do início do jejum, deve-se iniciar infusão venosa de solução glicosada a $5 \%$ a $100 \mathrm{ml} / \mathrm{h}$. A medida da glicemia capilar deve ser realizada a cada hora para monitorização adequada. Bolus de insulina regular podem ser usados caso ocorram elevações glicêmicas significativas.

Pacientes em uso de insulina poderão ser controladas com insulinoterapia venosa contínua (indicada para pacientes com maior labilidade glicêmica), ou sob regime de controle intermitente com insulina regular.

Para o dia do parto: $1 / 3$ da dose total de insulina usada na gestação; soro glicosado a 10\%,via intravenosa, 40 gotas/ minuto, glicemia capilar de $4 / 4$ horas e reposição com insulina regular, se necessário ${ }^{17}(\mathbf{D})$.

Após o parto, com a retirada da placenta, as alterações metabólicas são revertidas, e os níveis glicêmicos normalizados. $\bigcirc$ aleitamento materno deve ser estimulado ${ }^{5,23}(\mathbf{D})$.

\section{Cuidados e orientações em longo prazo}

Pacientes que desenvolvem DMG têm alto risco de recorrência em gestações futuras ${ }^{24}(\mathbf{B})$. Estas pacientes apresentam também risco de 20\% a 40\% de desenvolverem DM tipo 2, num período de 10 a $20 \operatorname{anos}^{5,23}$ (D).

Em nosso meio, observou-se que $44,8 \%$ das portadoras de DMG desenvolveram DM tipo 2 após 12 anos da gestação-índice ${ }^{25}(\mathbf{A})$.

Além das complicações no pós-parto imediato, estudos demonstraram que fetos macrossômicos têm risco aumentado de desenvolverem obesidade e DM durante a adolescência $^{26}(\mathbf{A})$.

É recomendado que a parturiente realize TOTG com $75 \mathrm{~g}$ de dextrosol após seis a oito semanas do parto.

Orientações gerais sobre melhoria dos hábitos de vida com estímulo à alimentação mais saudável, perda de peso e realização de atividade física regular devem ser adotadas como medidas preventivas.

\footnotetext{
Referências

I. Brody SC, Harris R, Lohr K. Screening for gestational diabetes: a summary of the evidence for the U.S. Preventive Services Task Force. Obstet Gynecol. 2003; 101:380-92.

2. Schmidt MI, Duncan BB, Reichelt AJ, Branchtein L, Matos MC, Costa e Forti A, et al. Gestational diabetes mellitus diagnosed with a 2-h 75-g oral glucose tolerance test and adverse pregnancy outcomes. Diabetes Care. 200I;
} 24: | I 5 |-5. 
3. Yang X, Hsu-Hage B, Zhang H, Zhang C, Zhang Y, Zhang C. Women with impaired glucose tolerance during pregnancy have significantly poor pregnancy outcomes. Diabetes Care. 2002;25: 1619-24.

4. Pettitt DJ. The 75-g oral glucose tolerance test in pregnancy. Diabetes Care. $2001 ; 24: 1129$

5. American Diabetes Association. Gestational diabetes mellitus. Diabetes Care. 2004;27(Suppl I):S88-90.

6. Jones CW. Gestational diabetes and its impact on the neonate. Neonata Netw. $2001 ; 20: 17-23$

7. Rudge MVC, Calderon IMP, Ramos MD, Maestá I, Souza LMS, Peraçoli JC. Perspectiva perinatal decorrente do rígido controle pré-natal em gestações complicadas pelo diabete. Rev Bras Ginecol Obstet. 1995; 17:26-32.

8. Brasil. Ministério da Saúde. Pré-natal de baixo risco (Manual técnico). Brasília (DF): Ministério da Saúde; 2000.

9. Reichelt AJ, Spichler ER, Branchtein L, Nucci LB, Franco LJ, Schmidt MI. Fasting plasma glucose is a useful test for the detection of gestational diabetes. Brazilian Study of Gestational Diabetes (EBDG) Working Group. Diabetes Care. 1998;21: | 246-9.

10. Rudge MVC, Calderon IMP, Ramos MD, Brasil MAM, Peraçoli JC. Comparação de dois métodos de rastreamento do diabete na gestação. Rev Bras Ginecol Obstet. | 994; | 6:203-5

I I. Ayach W, Calderon IMP, Rudge MVC, Costa RAA. Associação glicemia de jejum e fatores de risco como teste para rastreamento do diabete gestacional. Rev Bras Saúde Matern Infant. 2005;5:329-35.

12. Hanna FW, Peters JR. Screening for gestational diabetes; past, present and future. Diabet Med. 2002; 19:35।-8

13. Backx Cl, Lotgering FK, Cornelis H, Wallenburg S. Oral glucose tolerance test is a poor predictor of hyperglycemia during pregnancy. J Perinat Med. 1989; 17:253-7.

4. Rudge MV, Peracoli JC, Berezowski AT, Calderon IM, Brasil MA. The ora glucose tolerance test is a poor predictor of hyperglycemia during pregancy. Braz J Med Biol Res. 1990;23: 1079-89.

15. Rudge MVC, Calderon IMP, Ramos MD, Suetake H, Peraçoli JC. Inves tigação diagnóstica do diabetes na gestação. Rev Bras Ginecol Obstet. 1996; | $8: 2 \mid-6$
16. Rudge MV, Calderon IM, Ramos MD, Abbade JF, Rugolo LM. Perinata outcome of pregnancies complicated by diabetes and by maternal daily hyperglycemia not related to diabetes. A retrospective 10-year analysis. Gynecol Obstet Invest. 2000;50: 108-12.

17. Diabete e hipertensão na gravidez: manual de orientação. FEBRASGO Rudge MVC, Amaral MJ, editores. São Paulo: Federação Brasileira das Associações de Ginecologia e Obstetrícia; 2004. v.3.

18. Giuffrida FMA, Castro AA, Atallah A, Dib SA. Diet plus insulin compared to diet alone in the treatment of gestational diabetes mellitus: a systematic review. Braz | Med Biol Res. 2003;36: I 297-300.

19. Franz MJ, Bantle JP, Beebe CA, Brunzell JD, Chiasson JL, Garg A, et al. Evidence-based nutrition principles and recommendations for the treatment and prevention of diabetes and related complications. Diabetes Care. 2002; 25: $148-98$

20. Vidaeff AC, Yeomans ER, Ramin SM. Gestational diabetes: a field of controversy. Obstet Gynecol Surv. 2003;58:759-69.

21. Garcia-Bournissen F, Feig DS, Koren G. Maternal-fetal transport of hypoglycaemic drugs. Clin Pharmacokinet. 2003;42:303-13.

22. Langer $O$, Conway DL, Berkus MD, Xenakis EM, Gonzales $O$. A comparison of glyburide and insulin in women with gestational diabetes mellitus. N Engl Med. 2000;343: I 134-8.

23. Joslin Diabetes Center and Joslin Clinic. Guideline for detection and management of diabetes in pregnancy. Comunication Department 2003;617732-2695.

24. MacNeill S, Dodds L, Hamilton DC, Armson BA, VandenHofM. Rates and risk factors for recurrence of gestational diabetes. Diabetes Care. 200 I;24:659-62.

25. Silva MR, Calderon IM, Gonçalves LC, Aragon FF, Padovani CR, Pimenta WP. Ocorrência de diabetes melito em mulheres com hiperglicemia em gestação prévia. Rev Saúde Pública. 2003;37:345-50.

26. Catalano PM, Kirwan JP, Haugel-de Mouzon S, King J. Gestational diabetes and insulin resistance: role in short- and longterm implications for mother and fetus. J Nutr. 2003; 133:1674S-83S.

O texto completo da Diretriz: Diabetes mellitus gestacional está disponível nos sites: www.projetodiretrizes.org.br e www.amb.org.br. 